\title{
Evidence of a halo formation mechanism in the Spallation Neutron Source linac
}

\author{
Dong-O Jeon* \\ Institute for Basic Science, Daejeon, Republic of Korea \\ (Received 7 January 2013; published 23 April 2013; corrected 16 May 2013)
}

\begin{abstract}
A new halo formation mechanism and its mitigation scheme [D. Jeon, J. Stovall, A. Aleksandrov, J. Wei, J. Staples, R. Keller, L. Young, H. Takeda, and S. Nath, Phys. Rev. ST Accel. Beams 5, 094201 (2002)] are verified experimentally through a series of emittance measurements performed during the drift tube linac tank 1 commissioning of the Spallation Neutron Source. This is a rare experiment evidence of a halo formation mechanism. As the simulation predicts, the emittance measurements clearly show a visible halo reduction as well as a significant rms emittance reduction when the proposed round beam optics is employed. The emittance measurement results are consistent with multiparticle simulations and also consistent with wire scanner results. These measurements serve as a valuable code benchmarking for a beam under an intense space charge effect.
\end{abstract}

DOI: 10.1103/PhysRevSTAB.16.040103

PACS numbers: 41.85.Si, 41.75.-i, 29.27.-a

\section{INTRODUCTION}

The Spallation Neutron Source (SNS) accelerator system is designed to accelerate intense proton beams to energy of $1-\mathrm{GeV}$, delivering more than $1.4 \mathrm{MW}$ (upgradeable to $2 \mathrm{MW}$ ) of beam power to the neutron production target [1]. The peak current in the linac is $38 \mathrm{~mA}$ and the macropulse average current is $26 \mathrm{~mA}$ due to chopping. The SNS linac has the following structure; ion source, low-energy beam-transport (LEBT), radio-frequency quadrupole (RFQ), medium-energy beam-transport (MEBT), drift tube linac (DTL), coupled cavity linac (CCL), and superconducting linac. A primary concern is potential damage and radio activation of accelerator components resulting from uncontrolled beam losses. A major source of loss is beam halo that intercepts the bore of the linac. Many halo generation mechanisms have been studied such as space charge coupling resonances [2], mismatch [3], envelope instability [4], etc. Recently it is discovered that a fourth order space charge resonance $4 \sigma=360^{\circ}$ is excited in high intensity linear accelerators, practically replacing the envelope instability when depressed phase advance per cell $\sigma$ is close to $90^{\circ}$ [5] and experimentally confirmed [6].

Beam dynamics simulations of the SNS linac showed that the beam halo develops at low energy, but some halo particles survive acceleration to higher energies before being lost primarily on the CCL bore. This particle loss at higher energies results in radio activation of the CCL. A new halo generation mechanism was reported in the nonperiodic lattices such as the SNS linac MEBT (between RFQ and DTL) [7]. The nonlinear space charge force resulting from large transverse beam eccentricity $\sim 2: 1$ in the 1.6-m-long MEBT chopper section shown in the upper plot of Fig. 1 is responsible for halo formation. We call this particular MEBT optics "nominal beam optics."

It suppresses this kind of halo generation to design lattices with transverse beam eccentricity close to 1:1 in the two long drift sections as shown in the lower plot of Fig. 1. We call this MEBT optics "round beam optics." Multiparticle simulations show that halo and the rms

\footnotetext{
*jeond@ibs.re.kr

Published by the American Physical Society under the terms of the Creative Commons Attribution 3.0 License. Further distribution of this work must maintain attribution to the author(s) and the published article's title, journal citation, and DOI.
}

1098-4402/13/16(4)/040103(10) 


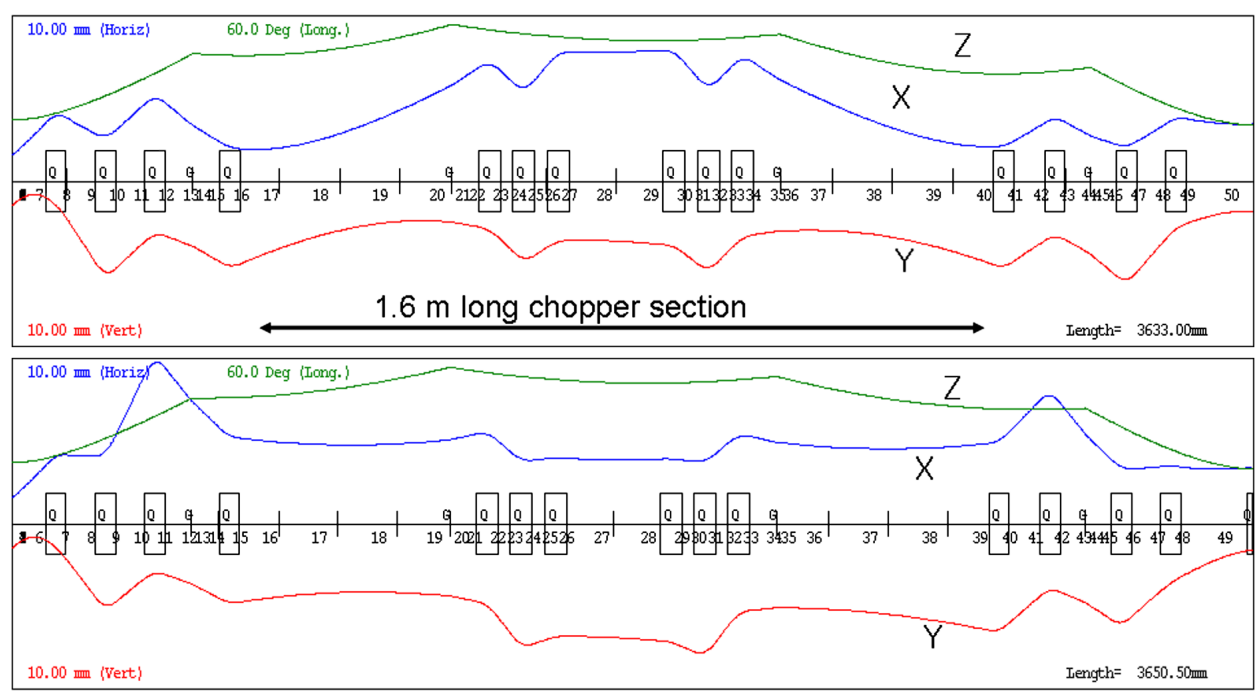

FIG. 1. MEBT beam profiles obtained from TRACE3D for the nominal beam optics in the upper plot and those for the round beam optics in the lower plot. These two sets of optics were employed for the emittance measurements. Plotted are $\sqrt{5} \sigma$ of beam size for each plane. The beam goes from left to right. There is RFQ upstream (on the left) and DTL tank 1 downstream (on the right).
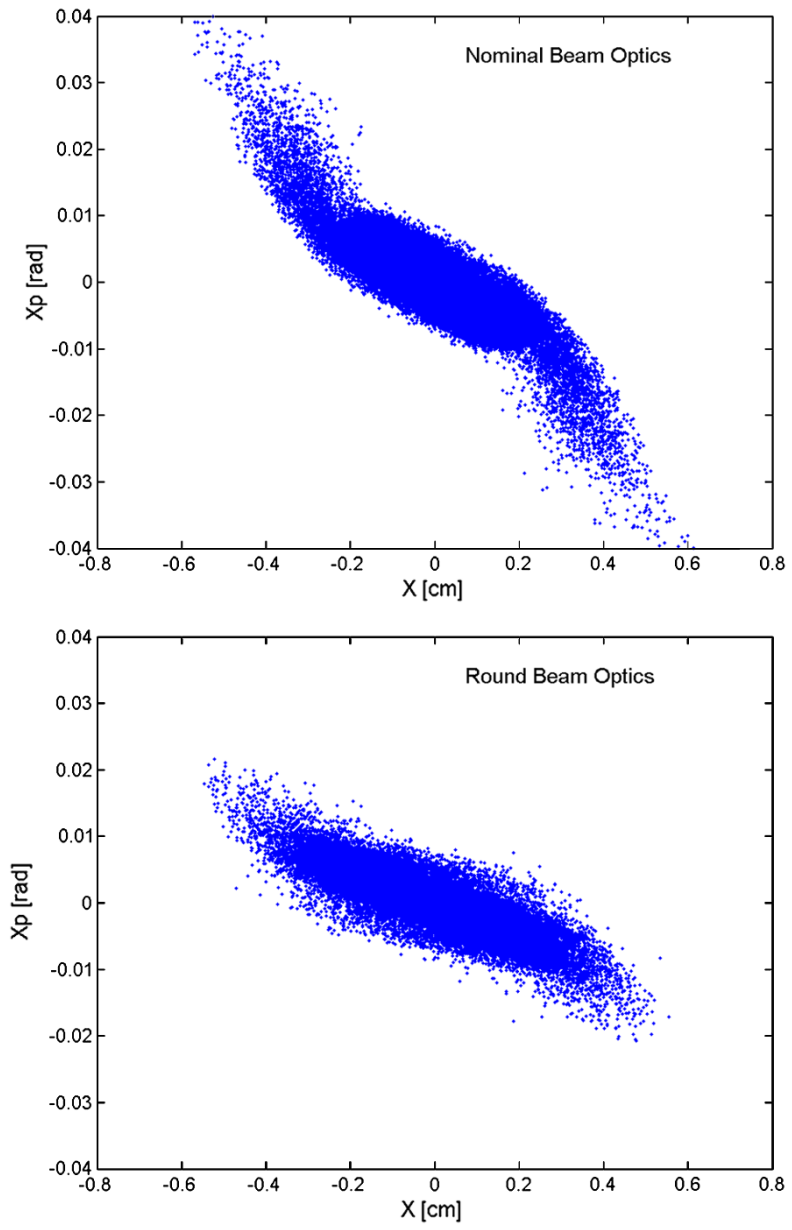

FIG. 2. Horizontal phase space plots of the nominal MEBT optics (the upper plot) and the round beam optics (the lower plot) at the MEBT exit. These plots are generated by simulations. This shows that round beam optics clearly reduces halo formation. In the emittance measurement, the halo thus generated for the nominal beam optics is observed in the top plot of Fig. 3. 
emittance in both planes are reduced significantly when the round beam optics is employed. Figure 2 shows the simulated beam distributions at the MEBT exit for the two different MEBT optics sets, clearly showing reduction of halo by the round beam optics. Modifying the MEBT optics and introducing adjustable collimators in the MEBT chopper target significantly reduce beam losses in the CCL, which is a preferred halo mitigation scheme. For the details, please refer to the previous study [7].

It is worth pointing out that beam sizes of the round beam optics are larger than those of nominal beam optics in general. So nonlinear effects of quadrupoles would be greater for the round beam optics, if this is the result of nonlinear field effects of quadrupoles.

The SNS DTL tank 1 commissioning is a rare opportunity to verify experimentally the validity of proposed halo generation mechanism and its mitigation schemes through emittance measurements unequivocally. In Sec. II experimental results are presented verifying the new halo generation mechanism and the effectiveness of the round beam optics. Then the conclusion follows in Sec. III. The study results are a rare verification of the halo formation mechanism through direct emittance measurements and serve as an important benchmarking of halo formation of high intensity beams.

\section{EXPERIMENTAL VERIFICATION OF THE HALO FORMATION MECHANISM}

Horizontal and vertical emittance measurements are performed after the DTL tank 1 at $Z=8.492 \mathrm{~m}$ in Fig. 9 to see if the proposed round beam MEBT optics reduces halo and rms emittance. The MEBT is located between $Z=0 \mathrm{~m}$ and $Z=3.650 \mathrm{~m}$, DTL tank 1 between $Z=3.650 \mathrm{~m}$ and $Z=7.803 \mathrm{~m}$. First, emittance measurements are done using the nominal MEBT optics. Then we adopt the round beam optics as shown in the lower plot of Fig. 1. Before measuring the emittance after the drift tube linac (DTL) tank 1, matching the MEBT to DTL tank 1 is performed by minimizing the measured rms emittance in both planes [8]. It is vital to remove potential mismatch between the MEBT and DTL tank 1. It should be pointed out that this particular round beam optics is slightly different in the middle of MEBT from that presented in the previous paper [7]. There are many sets of possible round beam optics and the one used in this study is one of them.

For the DTL tank 1 commissioning, a dedicated "diagnostics-plate" [9] is attached at the end of DTL tank 1 which is equipped with a variety of diagnostics devices including emittance scanners and wire scanners. Emittance scanners consist of horizontal and vertical emittance slits and harps installed, enabling emittance measurements in both planes. Emittance is measured with slits and 32-wire harps, taking data at every one microsecond. In the analysis, one chooses a narrow time window where data become stabilized.

Figure 3 shows the results of horizontal emittance measurements for two different MEBT optics sets; the nominal beam optics and the round beam optics. Compared with the nominal MEBT optics, the halo is visibly reduced and the rms emittance is significantly reduced from 0.454 to $0.289 \mathrm{~mm}$ mrad when the proposed round beam optics is employed. This confirms experimentally the halo generation mechanism identified in the previous paper [7]. It should be noted that substantial halo is generated mainly in the horizontal plane for the nominal beam optics even though MEBT is matched to DTL in the sense of minimizing rms emittances. Because there is only one electromagnetic quadrupole between the downstream end of DTL tank 1 and the emittance slits, the beam distribution is rather divergent in the horizontal plane. Figure 4 shows the results of the vertical emittance measurements. Again visible reduction in the halo is observed. The rms emittance is also reduced from 0.472 to $0.306 \mathrm{~mm}$ mrad. Figure 5 shows plots of normalized rms emittance vs clipping threshold. Emittance with threshold 0.01 is the rms emittance when the phase space area with intensity below 0.01 of maximum intensity is removed. This indicates that a significant contribution to the rms emittance comes from the low intensity halo for the nominal beam optics, contrary to the round beam optics. 

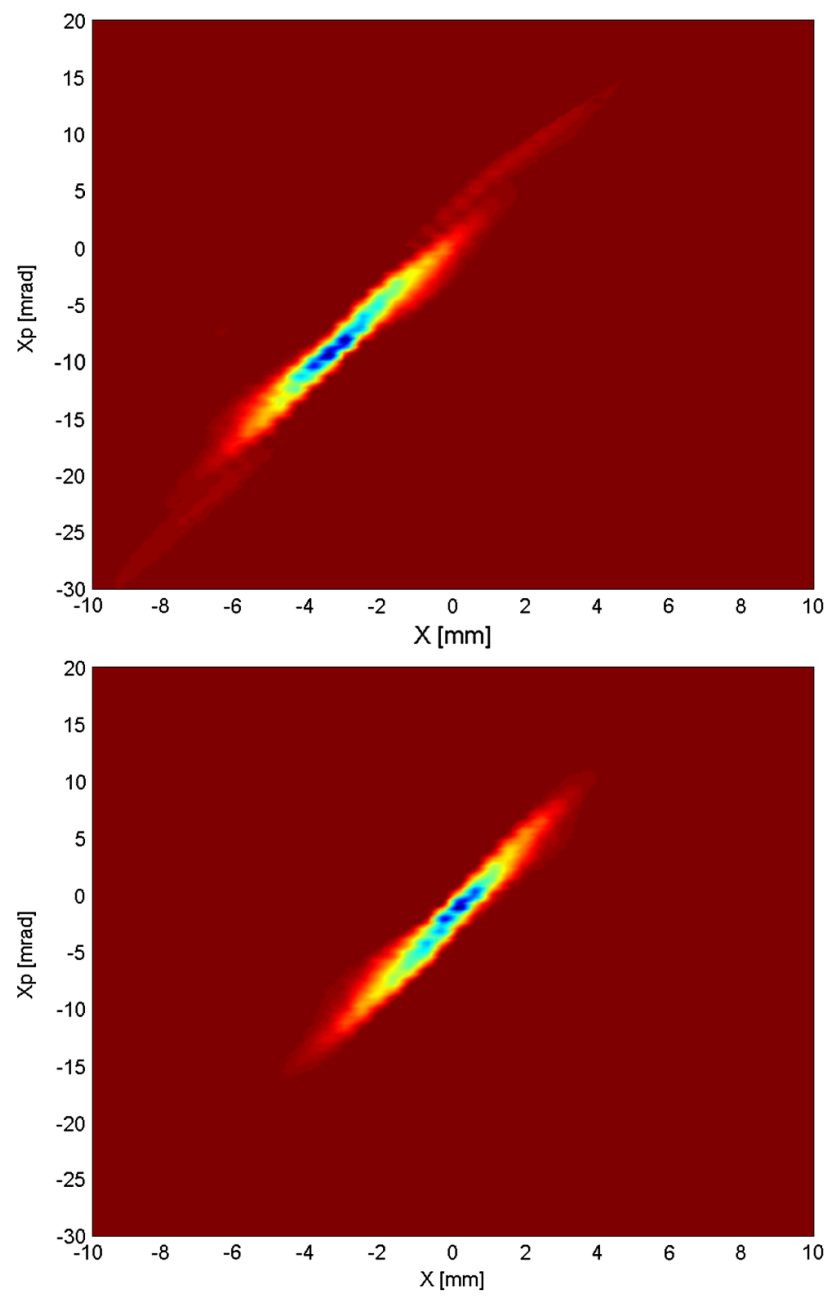

FIG. 3. Measured horizontal beam distribution in phase space of the nominal MEBT optics (the upper plot) and the round beam optics (the lower plot). The halo is visibly reduced, especially extended wings in the top plot. The rms emittance is $0.454 \mathrm{~mm}$ mrad for the upper plot and $0.289 \mathrm{~mm}$ mrad for the lower plot.

These measurement results showing significant reduction both in rms emittance and halo are consistent with the multiparticle simulations using the PARMILA code [10]. It is evident that the multiparticle code with space charge routine is adequately simulating the physics process involved. Beam distribution plots obtained from the simulation for the two MEBT optics sets are shown in Fig. 6. The upper plots are simulated beam distributions when the nominal MEBT optics is used and the lower plots when the round beam optics is used. The simulation predicts that the extended halo in the horizontal plane for the nominal beam optics disappears when the round beam optics is used, just like the measurement results for the round beam optics in Fig. 3. The simulation also predicts that the rms emittance in both planes is reduced significantly when the round beam optics is employed. The ratio of $\varepsilon_{x}$ (round beam optics)/ $\varepsilon_{x}$ (nominal beam optics $)=64 \%$ for measurements and $60 \%$ for simulations. Likewise $\varepsilon_{y}($ round beam optics $) / \varepsilon_{y}$ (nominal beam optics $)=66 \%$ for measurements and $88 \%$ for simulations. This is listed in Table I.

It is worth pointing out that in the vertical plane, measured emittance $\varepsilon_{y}$ decreases further for the round beam optics than the simulation. Coupling between $x$ and $y$ planes might be a possibility, because $\varepsilon_{y}$ decreases to $88 \%$ for the round beam optics in simulations. Further decrease may be attributed to our lack of knowledge of the initial beam distribution. SNS, LBNL, and LANL staff used the best available input beam distribution due to restrictions in 

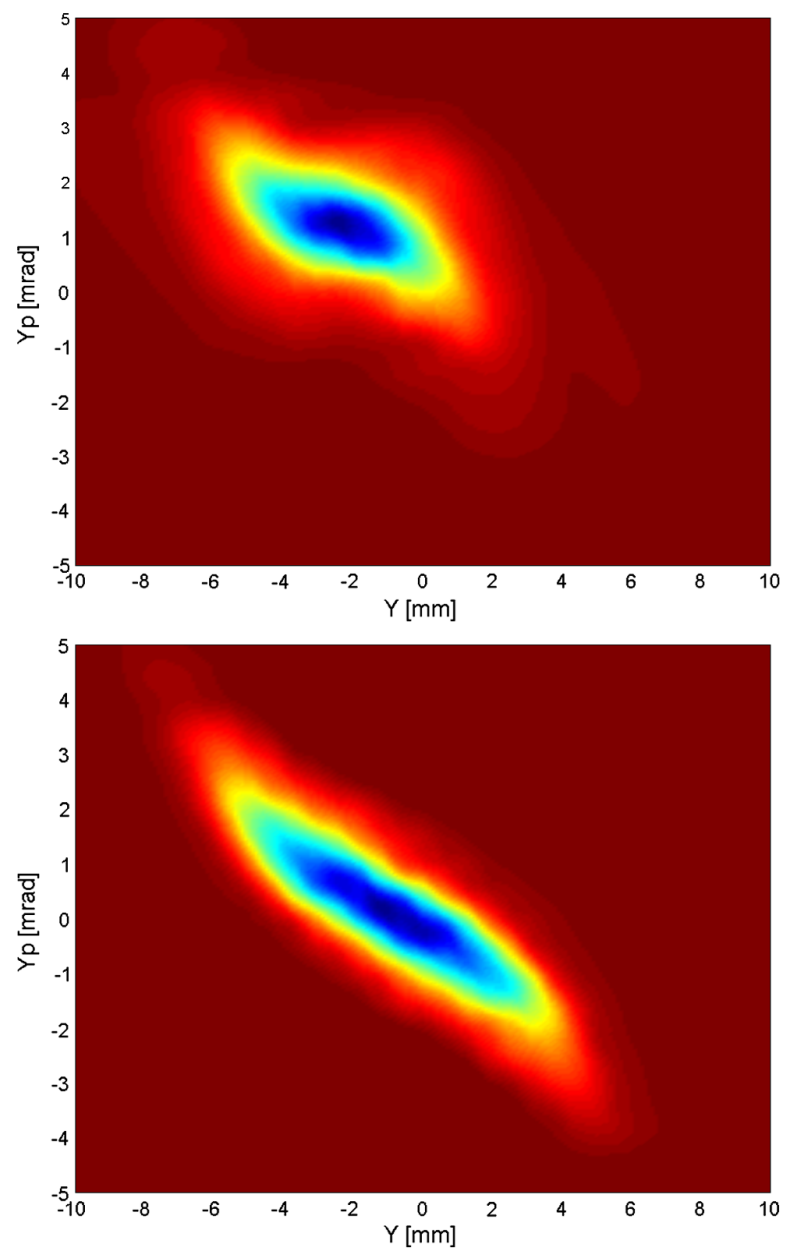

FIG. 4. Measured vertical beam distribution in phase space of the nominal MEBT optics (the upper plot) and the round beam optics (the lower plot). The halo is substantially reduced. The rms emittance is $0.472 \mathrm{~mm}$ mrad for the upper plot and $0.306 \mathrm{~mm}$ mrad for the lower plot.

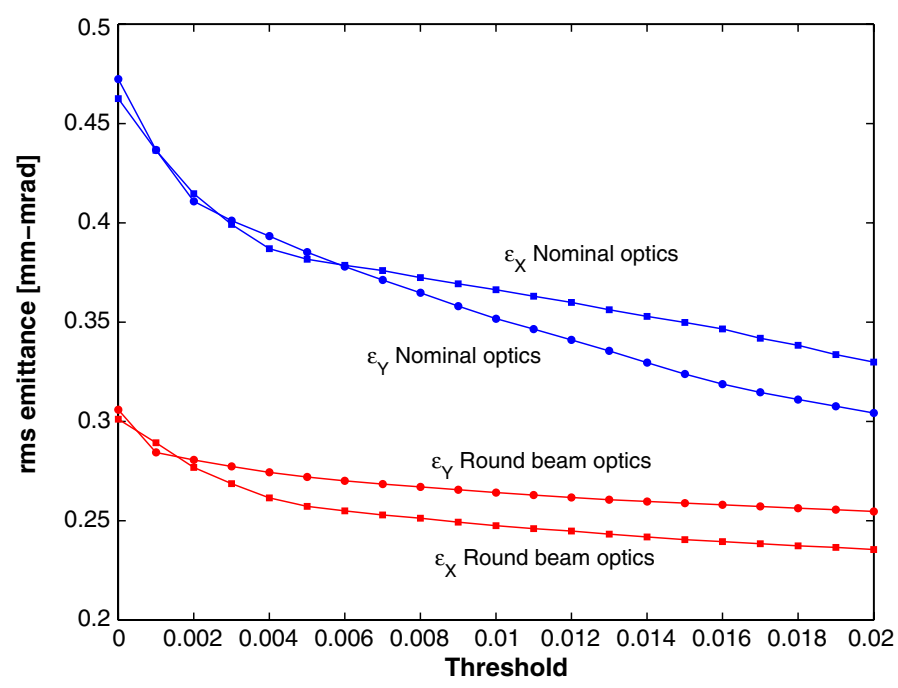

FIG. 5. Normalized rms emittance vs clipping threshold. Emittance value with threshold 0.01 means the rms emittance when the phase space area with intensity below 0.01 of maximum intensity is removed. It is clear that beam quality of round beam optics is far better than the nominal beam optics. 

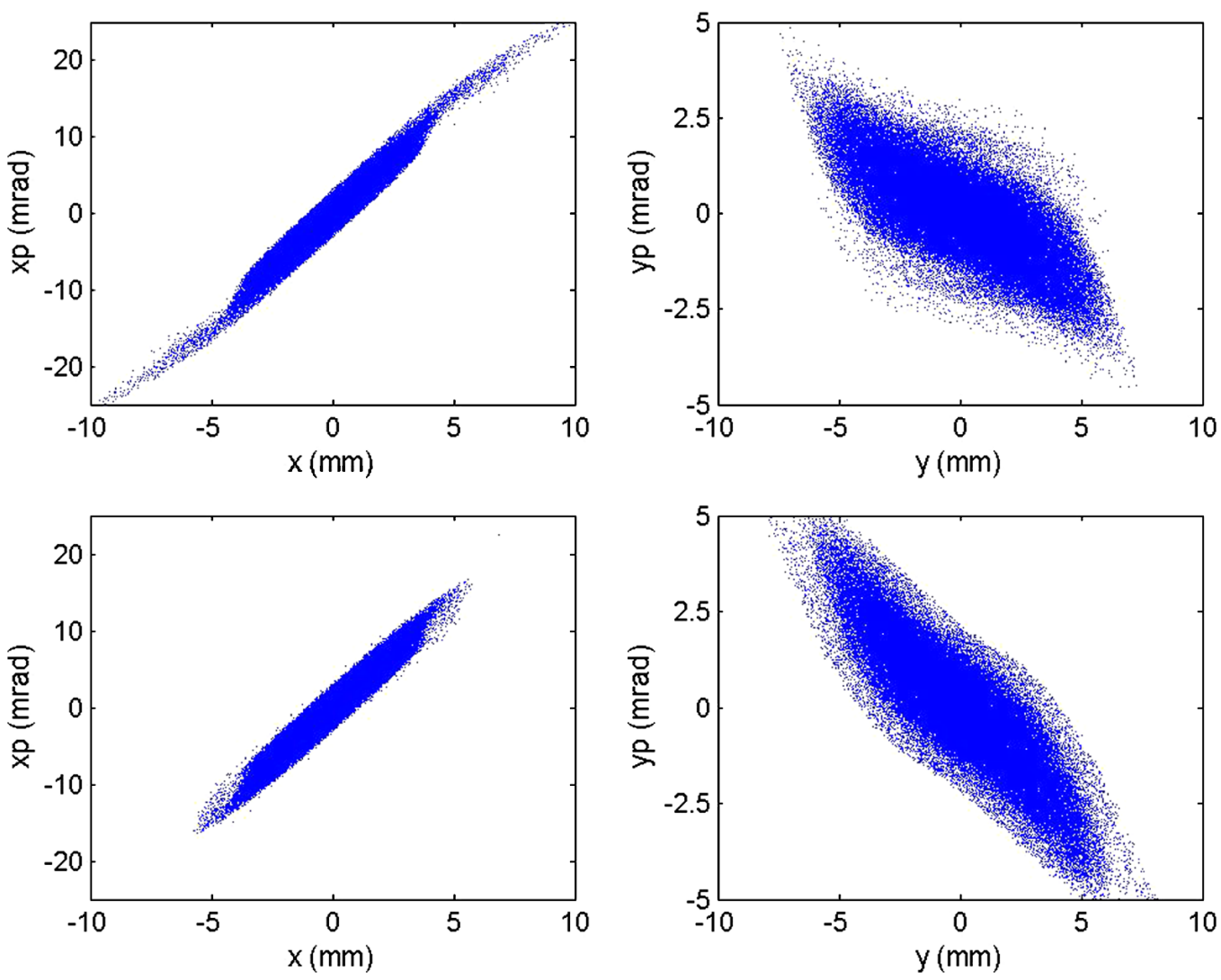

FIG. 6. Plots of simulated beam distributions for two different sets of MEBT optics. Upper plots are obtained using the nominal beam optics and lower plots using the round beam optics. Reduction in halo is visible in both planes, which is consistent with the measurement. These simulation plots should be compared with the measurements in Figs. 3 and 4.

available emittance measurements (this is well described in the Introduction and Fig. 1 of [7]). The input beam distribution the author used was obtained as follows: (i) reconstruct beam distribution based on the LEBT transverse emittance measurement before the RFQ; (ii) track the reconstructed beam distribution of step (i) through the RFQ using PARMTEQ code; (iii) longitudinal beam distribution was derived from the PARMTEQ simulation.

Figure 7 shows plots of measured beam profiles in red and blue curves for the two different sets of MEBT optics shown in Figs. 3 and 4, and simulated profiles in green curve for the nominal MEBT optics shown in the top plots of Fig. 6. These are height-normalized profiles obtained from projecting down the phase space distribution plotted in log scale. The measured profiles show that quite dense (a few percent level) and extensive halo has formed when the nominal MEBT optics is employed. However, the tail of measured beam profile is dramatically reduced when the round beam optics is employed. This suppression of beam tail for the round beam optics is quite consistent with the multiparticle simulations. It is worth noting that the extent of halo obtained from the simulation is consistent with that from the measurement,

TABLE I. Emittance ratio of two MEBT optics.

\begin{tabular}{lcc}
\hline \hline & Measurements & Simulations \\
\hline $\begin{array}{l}\varepsilon_{x}(\text { round beam }) / \varepsilon_{x} \\
(\text { nominal beam })\end{array}$ & $64 \%$ & $60 \%$ \\
$\begin{array}{l}\varepsilon_{y}(\text { round beam }) / \varepsilon_{y} \\
\text { (nominal beam })\end{array}$ & $66 \%$ & $88 \%$ \\
\hline \hline
\end{tabular}



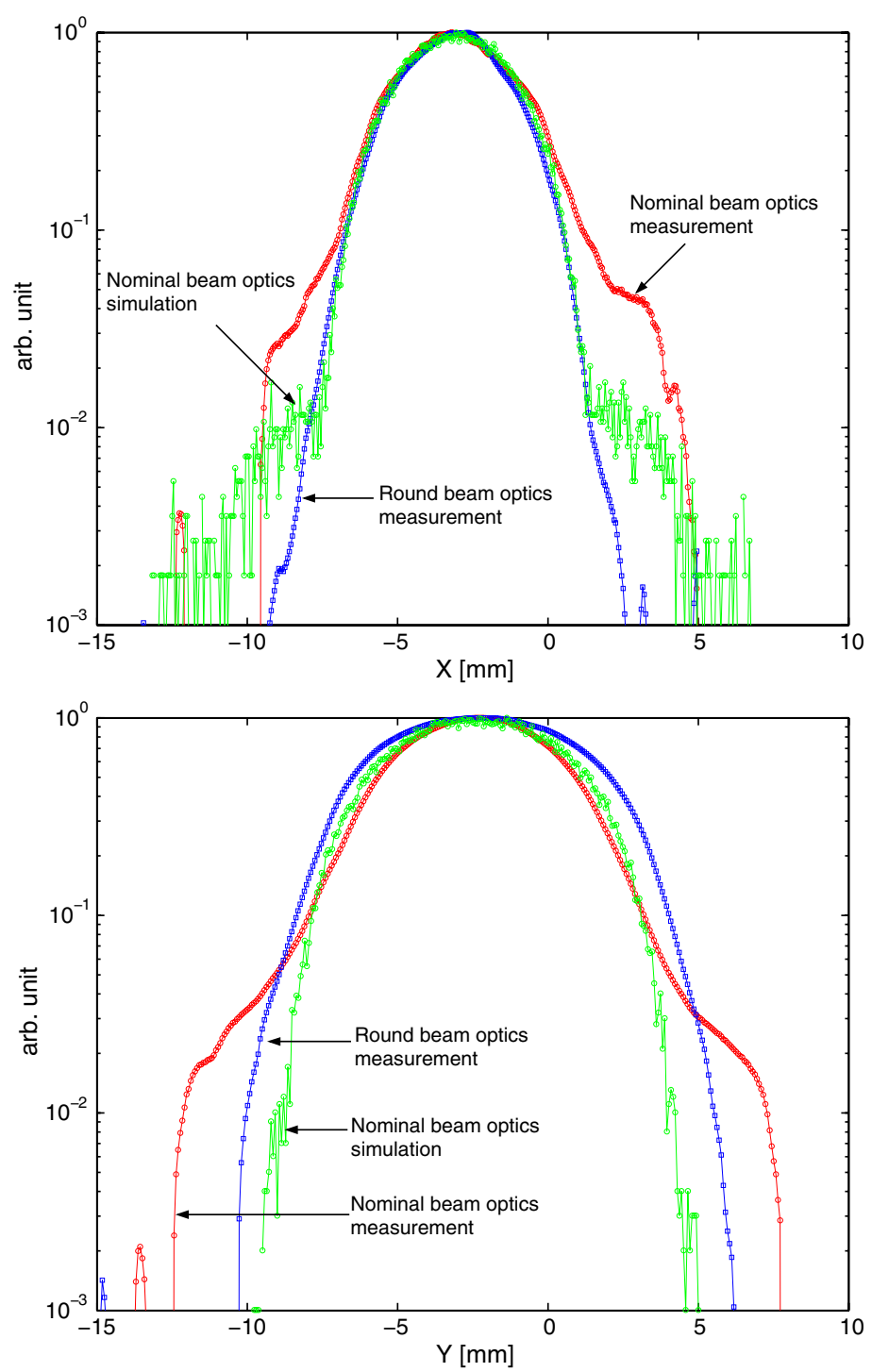

FIG. 7. Measured beam profile plots for two different sets of MEBT beam optics and simulated beam profile plot. Upper (lower) curves are horizontal (vertical) beam profiles.

which shows that the halo is bounded. More halos seem to form in reality than in the simulation as shown in the plots. However, there is a difference between the measured halo intensity and simulated halo intensity and this kind of detail depends on the accurate knowledge of initial beam distribution.

A comparison is made between the emittance data and raw wire scanner profile data. The beam profiles from the emittance data are consistent with the wire scanner beam profiles, as expected. The core of beam profiles obtained from the emittance data agrees well with the core wire scanner beam profiles for the two different sets of optics, as shown in Fig. 8. However, it is interesting to note that the wire scanner seems to overestimate the beam tail compared with the profile obtained from the emittance data.

A comparison of beam profile measurements with the models is performed to verify that actual MEBT optics is indeed consistent with the round beam optics shown in the bottom plot of Fig. 1. Figure 9 shows plots of horizontal and vertical rms beam sizes [mm] vs $Z$ position for the two MEBT optics sets. The top plots are for the nominal MEBT optics and the bottom plots for the round beam MEBT optics. The rms beam sizes obtained from wire scanners are plotted in 

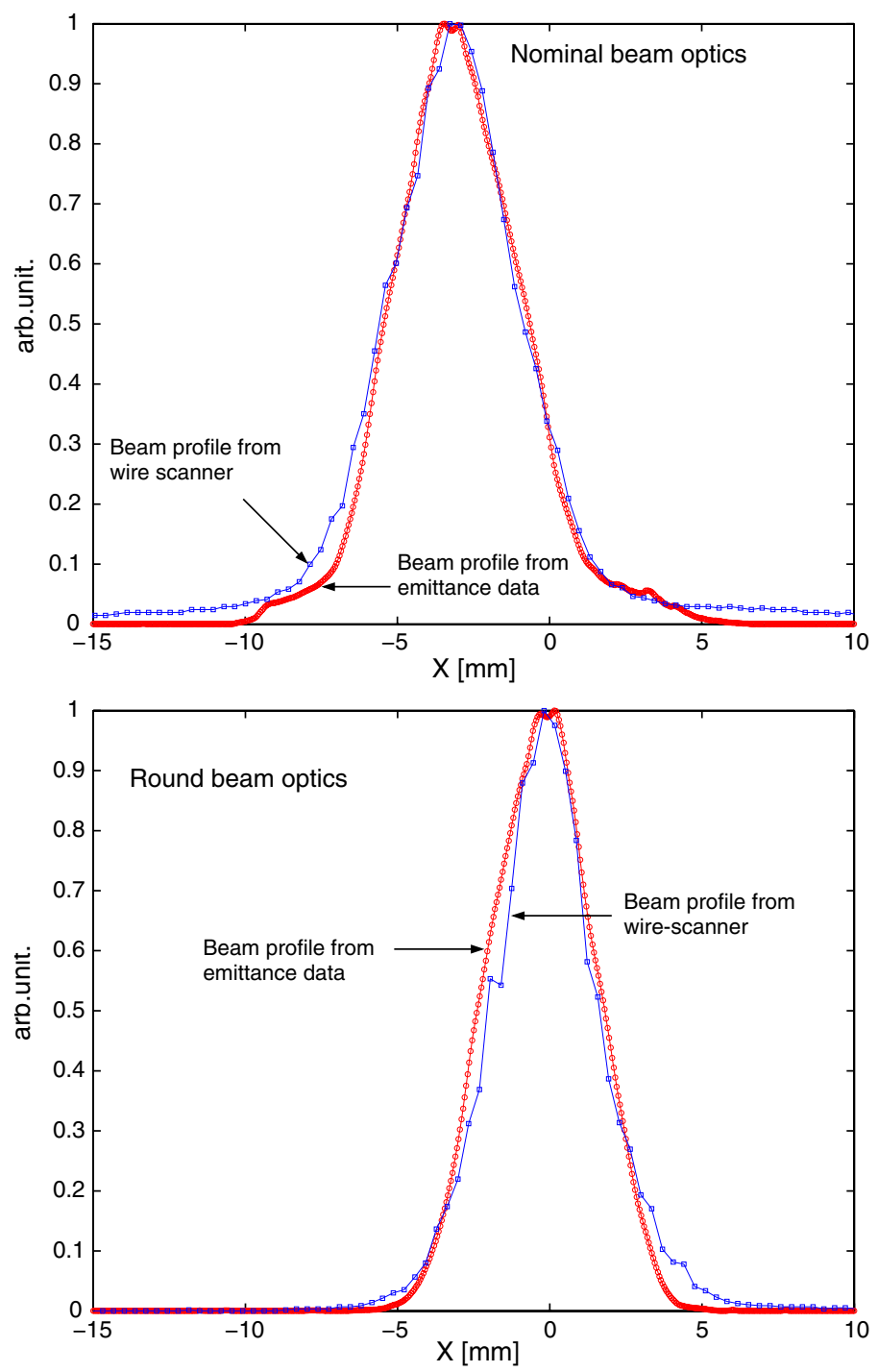

FIG. 8. Plots of horizontal beam profiles for two different MEBT optics sets. The top plot is for the nominal beam optics and the bottom plot for the round beam optics. Beam profile plots were obtained from the emittance data and wire scanner data. The wire scanner seems to overestimate the tail at low intensity.

solid squares and the rms beam sizes obtained from the TRACE3D code plotted in real lines. It is clear that the actual optics is consistent with the model in Fig. 1. There is a nice agreement between the beam profile data obtained from the wire scanner measurement and those from the TRACE3D code simulation.

It should be pointed out that there exists some uncertainty in our knowledge of the input beam distribution and the actual machine optics, thus introducing uncertainties to the quantitative comparison of rms emittance, beam profile, or Courant-Snyder parameters between the model and measurement. We observe appreciable variations over the time in the strength of four matching quadrupoles to obtain a proper matching, even though the current readings from the upstream ten quadrupoles are set for the same nominal beam optics. The gradient of the matching quadrupoles sometimes deviates from what the model predicts as much as $-2.3 \mathrm{~T} / \mathrm{m}$ for QH12 (horizontally focusing quadrupole in the MEBT) for the nominal beam optics as summarized in Table II. It is not clear how accurately the model optics reflects the actual optics of the accelerator. The detuning of the RFQ that happened during the commissioning also added uncertainties to the input beam parameters. 

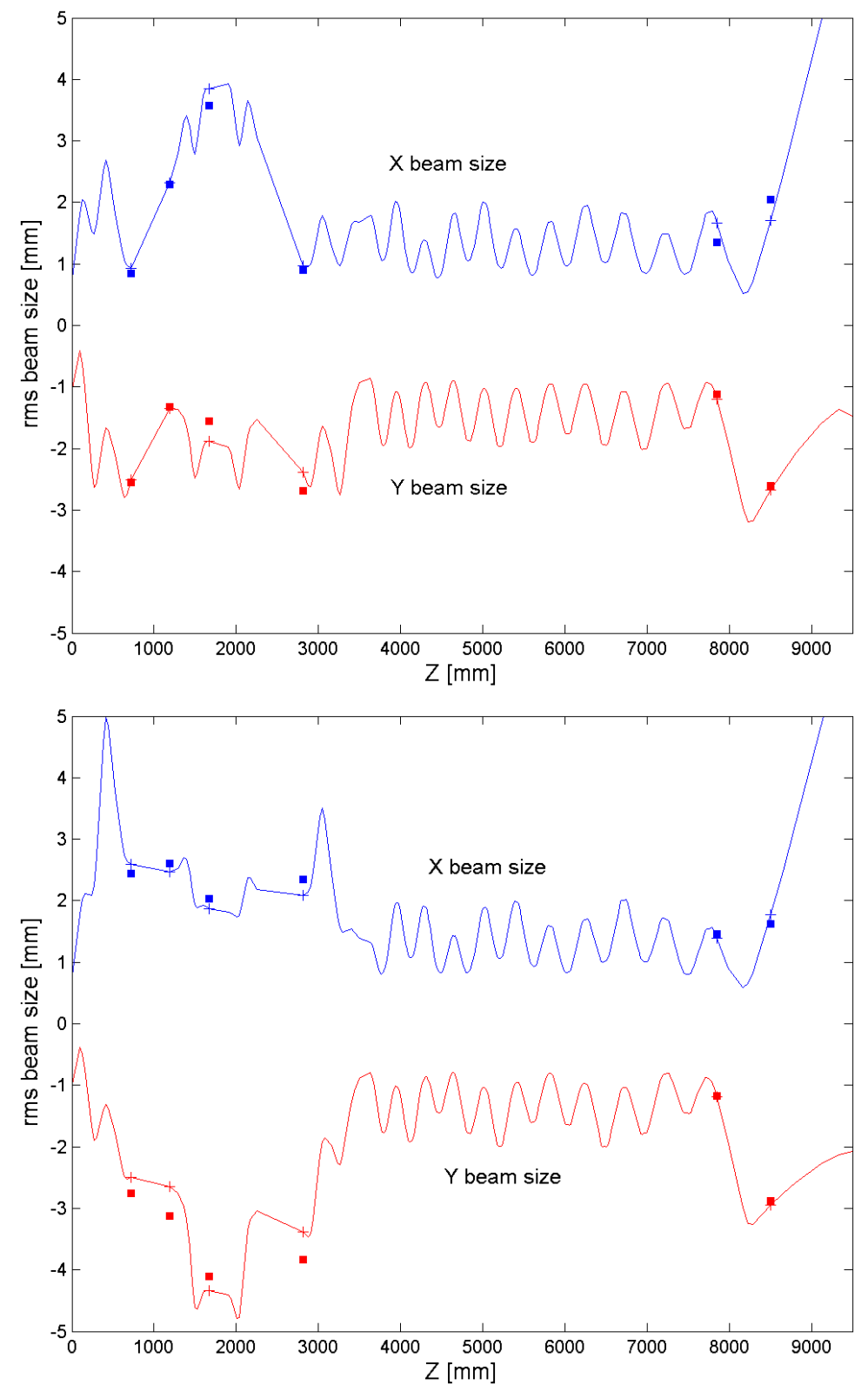

FIG. 9. Plots of horizontal and vertical $\mathrm{rms}$ beam sizes [mm] vs $Z$ position for two different MEBT optics. The top plots are for the nominal MEBT optics and the bottom plots for the round beam MEBT optics. The measured rms beam sizes from wire scanners are plotted in solid squares, those from TRACE3D in real lines. Horizontal and vertical emittance measurements are performed after the DTL tank 1 at $Z=8.492 \mathrm{~m}$. The MEBT is located between $Z=0 \mathrm{~m}$ and $Z=3.650 \mathrm{~m}$, DTL tank 1 between $Z=3.650 \mathrm{~m}$ and $Z=7.803 \mathrm{~m}$.

TABLE II. Matching quadrupole gradients for nominal beam optics.

\begin{tabular}{lccr}
\hline \hline & Design value $[\mathrm{T} / \mathrm{m}]$ & Before matching & After matching \\
\hline QV11 & 16.72 & 16.74 & 16.60 \\
QH12 & -25.96 & -26.12 & -28.31 \\
QV13 & 27.58 & 27.66 & 27.58 \\
QH14 & -18.50 & -17.31 & -16.86 \\
\hline \hline
\end{tabular}




\section{CONCLUSIONS}

In conclusion, emittance measurements verify the validity of the new halo formation mechanism in the MEBT and effectiveness of the round beam optics to mitigate halo formation. Emittance measurements confirm significant reduction in both the rms emittance and the halo when the round beam MEBT optics is employed. This also serves as a valuable benchmarking of beam dynamics codes with the space charge effect demonstrating that physics is adequately simulated.

\section{ACKNOWLEDGMENTS}

SNS is managed by UT-Battelle, LLC, under Contract No. DE-AC05-00OR22725 for the U.S. Department of Energy. Thanks also go to the Ministry of Science, ICT and Future Planning and the National Research Foundation of the Republic of Korea for their support under Contract No. 2011-0032011 for the data analysis. Special thanks go to S. Assadi and W. Blokland for their efforts on the emittance devices and wire scanners.

[1] J. Wei et al., in Proceedings of the 2001 Particle Accelerator Conference, Chicago, IL, 2001 (IEEE, New York, 2001), p. 319.

[2] D. Jeon, J. Holmes, V. Danilov, J. Galambos, and D. Olsen, Phys. Rev. E 60, 7479 (1999).

[3] J.-M. Lagniel et al., Nucl. Instrum. Methods Phys. Res., Sect. A 345, 46 (1994).

[4] S. Y. Lee and A. Riabko, Phys. Rev. E 51, 1609 (1995).

[5] D. Jeon, L. Groening, G. Franchetti, Phys. Rev. ST Accel. Beams 12, 054204 (2009).

[6] L. Groening, W. Barth, W. Bayer, G. Clemente, L. Dahl, P. Forck, P. Gerhard, I. Hofmann, M. S. Kaiser, M. Maier, S. Mickat, T. Milosic, D. Jeon, and D. Uriot, Phys. Rev. Lett. 102, 234801 (2009).

[7] D. Jeon, J. Stovall, A. Aleksandrov, J. Wei, J. Staples, R. Keller, L. Young, H. Takeda, and S. Nath, Phys. Rev. ST Accel. Beams 5, 094201 (2002).

[8] D. Jeon, C. M. Chu, J. Stovall, and S. Assadi, Nucl. Instrum. Methods Phys. Res., Sect. A 607, 517 (2009).

[9] M. Plum et al., in Proceedings of the 2001 Particle Accelerator Conference, Chicago, IL, 2001 (Ref. [1]), p. 2374.

[10] H. Takeda and J. Stovall, in Proceedings of the Particle Accelerator Conference, Dallas, TX, 1995 (IEEE, New York, 1995), p. 2364. 